\section{Topical Azithromycin 1\% in acute management of inflammatory pterygium}

\author{
Len V. Hua, Ami Halvorson, \\ Blair Lonsberry, Fraser Horn
}

Pacific University College of Optometry, Forest Grove, OR, USA

\section{Abstract}

The purpose of the article is to investigate the effects of topical azithromycin 1\% (Azasite) in treating acute ocular inflammation related to long standing pterygium. A prospective interventional study of 8 consecutive cases with pterygium presenting to our University Eye Clinics over the period of one month with acute symptoms of red eye(s) and irritation. After patient education and receipt of consent to the study, patients were treated with topical azithromycin 1\% (Azasite) twice a day for the first two days and once a day for the next 12 days. Anterior segment photo was taken before treatment and 3-day and/or 7-day after treatment in addition to eye examination.

Six out of the eight cases reported of significant improvement or resolution of signs and symptoms on the follow up visits. One severe case did not respond as well after a week of treatment and was successfully managed by topical prednisolone. Another case did not comply with the study protocol. In this small pilot study, Azasite was effective in management of acute mild to moderate ocular inflammation triggered by ocular surface disease, including pterygium. Severe ocular inflammation may require more potent anti-inflammatory therapy like topical steroids.

\section{Introduction}

Azithromycin, a semi-synthetic molecule derived from the macrolide erythromycin, is an antibiotic with broad-spectrum efficacy against gram-positive, gram-negative, and atypical pathogens. The structural modifications enhance cellular absorption and longer half-life as compared to other macrolides, which allow for wide clinical applications from respiratory to ophthalmological complications. ${ }^{1}$ Oral azithromycin has been used for decades to treat upper respiratory tract infection, otitis media, trachoma, and ocular toxoplasmosis. Recently, the United States Federal Drug Administration (FDA) approved topical azithromycin 1\% (Azasite) for treatment of bacterial conjunctivitis. ${ }^{2}$
In addition to its well-known antibiotic properties, azithromycin has been shown to exert anti-inflammatory effects. First evidence to suggest anti-inflammatory effects for azithromycin was found in patients with lung transplantation. As an adjunct therapy to immunosupression in lung transplantation, the lung lavage from some patients who improved in the course of recovery suffered from complications, had reduced infiltration of neutrophils and inflammatory cytokine interleukin-8 (IL8), suggesting possible inhibition of neutrophils recruitment by azithromycin. ${ }^{3}$ Further evidence was shown in inflammatory cells, azithromycin may downregulate the synthesis of pro-inflammatory cytokines and reduce the degradation of the accessory inhibitor of $\kappa B$ protein (IאB). ${ }^{4}$

Glucocorticoids are potent anti-inflammatory drugs widely used in medicine for various inflammatory diseases, including ophthalmic inflammations. The mechanisms of action for the anti-inflammatory activity of these drugs have been shown to inhibit expression of proinflammatory cytokines, inhibit phospholipase $\mathrm{A} 2$, and modulate immune functions in addition to NF- $\mathrm{KB}$ pathway involvement. ${ }^{5}$

One way to investigate the potential antiinflammatory effects of azithromycin is to compare its property to glucocorticoids. Hence, azithromycin's anti- inflammatory potency was recently measured using activated NF- $\kappa$ B assay on human lung carcinoma cell line (A549) in comparison with that of hydrocortisone and dexamethasone. Even without the mucoadhesive vehicle Durasite as in its topical formulation, azithromycin was shown to exert about 4 orders of magnitude weaker than that of hydrocortisone and thus confirming its antiinflammatory potential. ${ }^{6}$

Since its FDA approval in 2007, topical azithromycin has been used increasingly for ocular surface diseases, including blepharitis, a chronic inflammation of the eyelid. The pathophysiology of blepharitis is not fully elucidated, but bacterial colonization and inflammation are likely involved in the process similar to chronic lung infections. ${ }^{7}$ The effectiveness of topical azithromycin in blepharitis may be due to its dual anti-infective and antiinflammatory properties.

Pterygia are common ocular surface lesions arising from fibrovascular growth from the conjunctiva and extending onto the cornea. Chronic UV exposure has been implicated as one of the etiological factors. Patients with pterygia often experience recurrent inflammatory episodes that bring them to eye care professionals and are often managed by topical steroids, like loteprednol, prednisolone and steroid-antibiotic combination. ${ }^{8}$ Although, the definitive treatment is surgical excision of the pterygium, surgical intervention is not an option for many patients because of cost or
Correspondence: Len V. Hua, Pacific University College of Optometry, 2043 College Way, Forest Grove OR 97116 USA.

Tel. +1.503.352.3059 - Fax: +1.503.352.2929.

E-mail: lenvhua@pacificu.edu

Key words: azasite, tobradex, blepharitis, dry eye pinguecula, ocular surface disease.

Acknowledgments: the authors would like to thank Drs. Yudcovitch L, Mallette E, Overtone S, and Hamel $\mathrm{C}$ for their management of patients in the study.

Received for publication: 21 September 2011. Accepted for publication: 26 October 2011.

This work is licensed under a Creative Commons Attribution NonCommercial 3.0 License (CC BYNC 3.0).

(C) Copyright L.V. Hua, 2011

Licensee PAGEPress, Italy

Optometry Reports 2011; 1:e2

doi:10.4081/optometry.2011.e2

concerns, and postoperative recurrence can occur frustrating surgeons and patients alike. ${ }^{9}$

In this pilot study, the effect of topical azithromycin in acute management of inflammation was investigated with the hypothesis that the recurrent inflammatory events seen in patients with pterygium may be secondary to desiccate and abnormal ocular surface, and bacterial colonization, thus could respond beneficially from topical azithromycin.

\section{Materials and Methods}

Azithromycin ophthalmic solution (1\%, Inspire Pharmaceuticals, Inc., Raleigh, NC, USA) was used in the study. Pacific University Institution Review Board was consulted and approval was granted before the study.

Patients presented to the University Eye Clinics with inflamed pterygia were enrolled in the study after education on the study and consent was received. The inclusion criterion recruited adult patients with chief complaints of ocular irritations and redness secondary to pterygia in one or both eyes. The study excluded any patient who had active fungal/viral infection or recent ocular surface surgery within 90 days. Topical azithromycin was prescribed for twice a day for the first two days and once a day for the next 12 days in the affected eye(s). Patients were instructed on how to instill the medication properly. Anterior segment photography was taken prior to administration of topical azithromycin and at 3-day follow up and/or 7-day follow up exam. Patient history, symptom assessment and eval- 
uation of signs by slit lamp examination were performed at every visit by the same practitioner who examined the patients at the first visit. Conjunctival hyperemia was graded following the guide published by Efron et al. in 2001. ${ }^{10}$

\section{Results}

Eight patients, 5 females and 3 males from 36 to 58 years old with average age of 46 years old were recruited consecutively at our three University Eye Clinics over a period of one month (Table 1). The majority of the patients (6/8) had lived near the equator before immigrating to the United States (US). A few representative patients are presented to illustrate more details on the study.

Patient \#2: A 54-year-old Hispanic male complained of redness, irritation, crusts in the morning and occasional blurry vision in the left eye for the past month. His visual acuity (VA) was 20/20 OD, OS, OU without correction. Pupils and extraocular motility were normal. Anterior segment examination with slit lamp microscopy showed inflamed pterygium with grade 4 conjunctival hyperemia OS (Figure 1A), much greater than OD. Patient was given topical azithromycin to use twice day for first two days and once a day for the rest of the two weeks. On the follow up visit one week later, the patient reported feeling better with less redness, irritation and crustings. His VA was 20/20 and slit lamp examination showed less hyper-
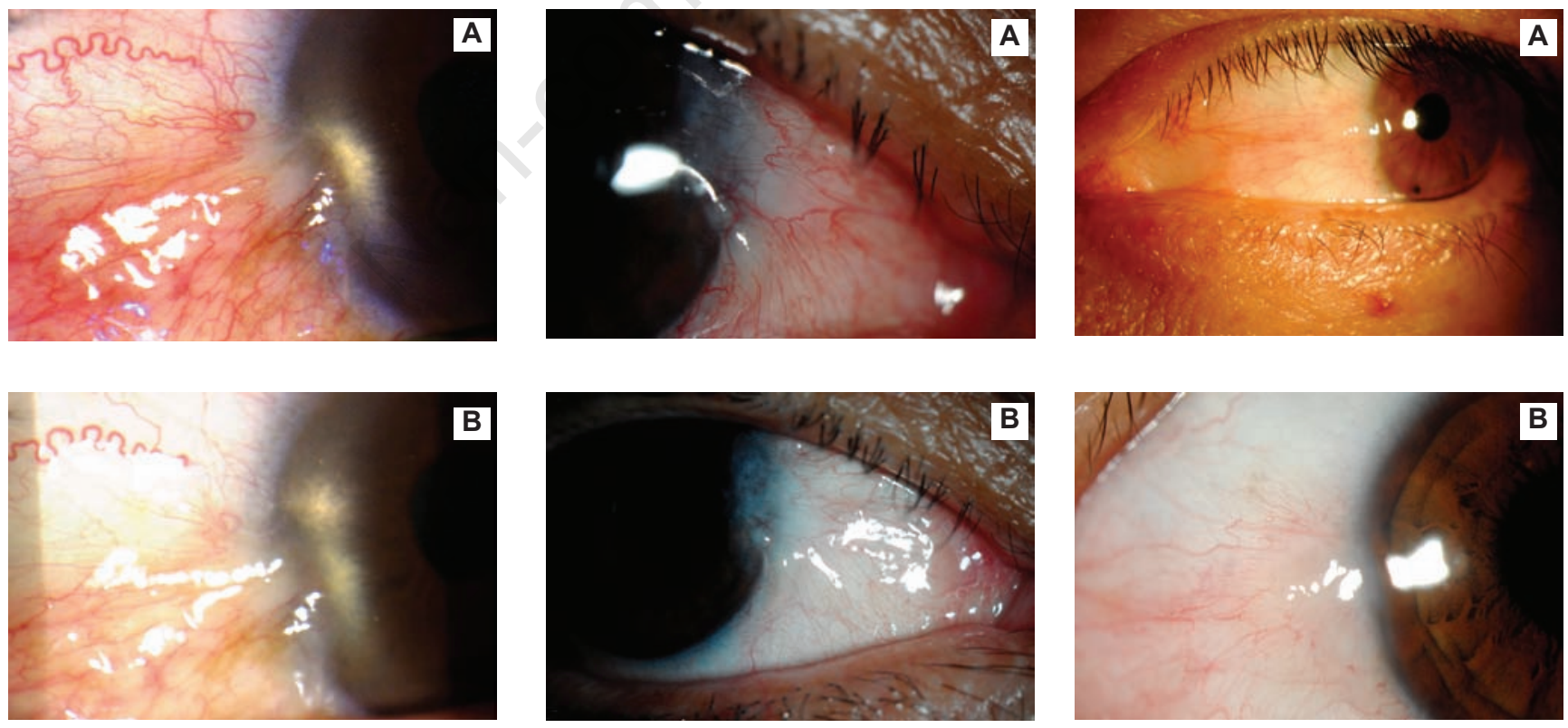

Figure 1. Bilateral inflamed pterygia. A) Moderate inflamed pterygium OS (patient \# 2); B) Resolving pterygium OS after 1week treatment with Azasite (Patient \# 2). emia (grade 2 conjunctival hyperemia) OS (Figure 1B). Patient was educated to continue taking topical azithromycin for another week and to maintain lid hygiene regimen for longterm management. Ultraviolet light protection and supplemental lubrication were also advised.

Patient \#4: A 58-year-old Hispanic female complained of redness, burning, tearing and irritation in both eyes for the last three months. Her ocular examination was unremarkable, except anterior segment examination with slit lamp microscopy showed inflamed pterygium OU (Figure 2A). Patient was given topical azithromycin to use twice day for first two days and once a day for the rest of the two weeks. On the follow up visit one week later, the patient did not feel better with the topical azithromycin and still complained of redness, burning, tearing and irritation in both eyes. Slit lamp examination showed grade 4 hyperemia OD and OS. Cornea was clear and there were no cells or flare in the

Table 1. Summary of study subjects.

\begin{tabular}{lccll} 
Case \# & Gender & Age & Sign and symptom relief & Comments \\
1 & $\mathrm{~F}$ & 36 & Less redness and irritation & \\
2 & $\mathrm{M}$ & 54 & Less redness and irritation & \\
\hline 3 & $\mathrm{~F}$ & 42 & Less redness and irritation & \\
4 & $\mathrm{~F}$ & 58 & No relief or improvement & Switch to topical prednisolone \\
\hline 5 & $\mathrm{M}$ & 37 & Less redness and irritation & \\
6 & $\mathrm{M}$ & 52 & No relief or improvement & Noncompliant \\
\hline 7 & $\mathrm{~F}$ & 39 & Less redness and irritation & \\
8 & $\mathrm{~F}$ & 52 & Less redness and irritation & \\
\hline
\end{tabular}

(IOP) was $13 \mathrm{mmHg}$ OD and OS. Patient was educated to discontinue taking topical azithromycin and start taking topical prednisolone (0.12\%, Pred Mild, Allergan, Inc., Irvine, CA, USA) for four times a day for one week. On the following visit a week later, she felt better with less redness and irritation. Slit lamp examination showed grade 1 conjunctival hyperemia OD and OS. Her IOP was $11 \mathrm{mmHg}$ OD and OS. Patient was educated to taper topical prednisolone to twice a day for a few more days and stop. Ultraviolet light protection, supplemental lubrication, and lid hygiene were also advised for long-term management.

Patient \#5: A 37-year-old Hispanic male complained of intermittent redness and irritation in both eyes for the last two years. Ocular examination was normal except anterior segment examination showed pterygium $\mathrm{OU}$ with $2+$ conjunctival hyperemia (Figure 3A). Patient was given topical azithromycin to use 
twice day for first two days and once a day for the rest of the two weeks. On the follow up visit 3 days later, the patient reported feeling better with less irritation and redness (grade 1 conjunctival hyperemia) (Figure 3B). Patient was educated to continue taking topical azithromycin for another week and to maintain lid hygiene regimen for long-term management. Ultraviolet light protection and supplemental lubrication were also emphasized.

In summary, six out of the eight patients reported and showed significant improvements in ocular sign (less hyperemia) and symptom (less burning or irritation) over the period of one week (Figure 1), a few appreciated the relief at 3-day follow up visit (Figure 3). One patient with severe ocular inflammation did not respond well to Azasite and was successfully managed with topical prednisolone (Figure 2). Another patient (\#6) was not compliant to the treatment.

\section{Discussion}

Pterygium is a common ocular surface disease that manifests as a fibrovascular conjunctival outgrowth encroaching onto the cornea. It can cause astigmatism, ocular irritations, dryness and unappealing cosmesis. ${ }^{11}$ The exact mechanisms underlying its pathogenesis is unclear, but it has been associated with various chronic stimuli such as ultraviolet (UV) irradiation, sawdust and wind exposure, and dry eye disease. ${ }^{12-14}$ These insults may trigger common inflammatory cascade mediated by nuclear factor kappa B (NF-кB) pathway which has recently been shown to be activated in pterygium. ${ }^{15}$

Topical azithromycin (1\%) is approved by FDA as a topical antibiotic for treatment of bacterial conjunctivitis, however it has been shown recently to relieve inflammatory sign and symptom of posterior blepharitis as measured by eyelid redness and meibomian gland blockade. ${ }^{16}$ Furthermore, 1\% azithromycin in DuraSite, a mucoadhesive vehicle, achieved high ocular surface tissue level. ${ }^{17}$ Azithromycin's anti-inflammatory properties have been shown to be mediated by its ability to inhibit pro-inflammatory NF- $\kappa \mathrm{B}$ activity in lung carcinoma cell line ${ }^{6}$ and to suppress zymosan-stimulated production of proinflammatory cytokines in human corneal epithelial cells. ${ }^{18}$ These data support the hypothesis that ocular surface disease whereby an inflammatory cascade mediated by activation of the NF$\kappa \mathrm{B}$ pathway, such as pterygium, may also be inhibited and relieved by topical azithromycin.

In the present study the majority (6/8) of our patients with acute inflammation secondary to pterygium responded beneficially with topical azithromycin treatment for one week, whereas severe manifestation (1/8) required topical steroid. This is the first case series to show the effectiveness of topical azithromycin in managing acute mild to moderate inflammation secondary to longstanding and progressive pterygium. Although steroids are frequently used to treat ocular inflammation, it is not always desirable as in children and steroid responders hence topical azithromycin can serve as another effective remedy. In addition, topical azithromycin can be used long term to maintain healthy ocular surface and minimize inflammatory recurrence.

The remarkable observation from the study is that most of the study subjects (6/8) had lived near the equator before immigrating to the US. This finding is in line with various epidemiologic studies indicating that ethnicity and/or geography is an independent predictor of pterygium prevalence varying from $2.9 \%$ in northern China to $31 \%$ in Lima, Peru (near the equator). ${ }^{19,20}$ Despite major advances in surgical intervention of pterygium, many patients are still suffered from the condition because of surgical cost and concerns, and postoperative recurrence can arise. ${ }^{9}$ Therefore, chronic medical managements are still in demand for many patients, especially low-income - patients with pterygium. Long-term maintenance of healthy ocular surface with lid hygiene and UV protection are crucial. Additionally, topical azithromycin can also be a part of the regimen for ongoing ocular surface care.

The limitation of this study is its small sample size and the lack of comparison groups, such as control or steroid. Future study with larger sample size and relevant comparison groups would provide a further support to Azasite's anti-inflammtory properties and effectiveness in management of inflamed pterygia.

In conclusion, this study provides the first evidence that topical azithromycin could be effective in clinical management of mild to moderate inflammation secondary to pterygium provoked by underlying chronic abnormalities of the ocular surface.

\section{References}

1. Wilms EB, Touw DJ, Heijerman HG. Pharmacokinetics of azithromycin in plasma, blood, polymorphonuclear neutrophils and sputum during long-term therapy in patients with cystic fibrosis. Ther Drug Monit 2006;28:219-25.

2. Kuper H, Wormald R. Topical azithromycin: New evidence. BJO 2007;91: 55667.

3. Verleden GM, Vanaudenaerde BM, Dupont LJ, Van Raemdonck DE. Azithromycin reduces airway neutrophilia and interleukin-8 in patients with bronchiolitis obliterans syndrome. Am J Respir Crit Care Med 2006;174:566-70.
4. Aghai ZH, Kode A, Saslow JG, et al. Azithromycin suppresses activation of nuclear factor- $\kappa \mathrm{B}$ and synthesis of proinflammatory cytokines in tracheal aspirate cells from premature infants. Pediatr Res 2007;62:483-8.

5. Schleimer RP. An overview of glucocorticoid anti-inflammatory actions. Eur J Clin Pharmacol 1993;45 Suppl 1:S3-7.

6. Cheung PS , Si EC, Hosseini K. Antiinflammatory Activity of Azithromycin as

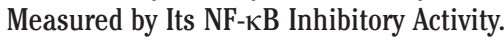
Ocul Immunol Inflamm 2010;18:32-7.

7. Gray GC, McPhate DC, Leinonen M, et al. Weekly oral azithromycin as prophylaxis for agents causing acute respiratory disease. Clin Infect Dis 1998;26:103-10.

8. Chui J, Coroneo MT, Tat LT, et al. Ophthalmic Pterygium: A Stem Cell Disorder with Premalignant Features. Am J Pathol 2011;178:817-27.

9. Pan HW, Zhong JX, Jing CX. Comparison of Fibrin Glue versus Suture for Conjunctival Autografting in Pterygium Surgery: A Meta-Analysis. Ophthalmology 2011;118: 1049-54.

10. Efron N, Morgan PB, Katsara SS. Validation of grading scales for contact lens complications. Ophthalmic Physiol Opt 2001;21:17-29.

11. Gazzard G, Saw SM, Farook M, et al. Pterygium in Indonesia: prevalence, severity and risk factors. Br J Ophthalmol 2002; 86:1341-6.

12. Moran DJ, Hollows FC. Pterygium and ultraviolet radiation: a positive correlation. Br J Ophthalmol 1984;68:343-6.

13. Detels R, Dhir SP. Pterygium: a geographical study. Arch Ophthalmol 1967;78: 48591.

14. Lekhanont K, Rojanaporn D, Chuck RS, Vongthongsri A. Prevalence of dry eye in Bangkok, Thailand. Cornea 2006;25: 11627.

15. Siak JJ, Ng SL, Seet LF, et al. The nuclearfactor $\kappa \mathrm{B}$ pathway is activated in pterygium. Invest Ophthalmol Vis Sci 2011;52: 230-6.

16. Luchs J. Efficacy of topical azithromycin ophthalmic solution intreatment of posterior blepharitis. Adv Ther 2008;25:858-70.

17. Bowman LM, Si E, Pang J, et al. Development of a topical polymeric mucoadhesive ocular delivery system for azithromycin. J Ocul Pharmacol Ther 2009;25:133-9.

18. Li DQ, Zhou N, Zhang L, et al. Suppressive Effects of Azithromycin on ZymosanInduced Production of Proinflammatory Mediators by Human Corneal Epithelial Cells. Invest Ophthalmol Vis Sci 2010; 51:5623-9.

19. Rojas J, Ma'laga H. Pterygium in Lima, Peru. Ann Ophthalmol 1986;18:147-9.

20. Ma K, Xu L, Jie Y, et al. Prevalence of and factors associated with pterygium in adult Chinese: the Beijing Eye Study. Cornea 2007;26:1184-6. 